\title{
Suboccipital trans-horizontal fissure approach for cerebellar hemorrhage with rupture into the upper fourth and third ventricles: the first clinical experience. Illustrative cases
}

\author{
Ryota Tamura, MD, PhD, ${ }^{1,2}$ Makoto Katayama, $\mathrm{MD}, \mathrm{PhD},{ }^{2}$ Yuki Kuranari, $\mathrm{MD}, \mathrm{PhD},{ }^{2}$ and Takashi Horiguchi, MD, $\mathrm{PhD}{ }^{1}$ \\ ${ }^{1}$ Department of Neurosurgery, Keio University School of Medicine, Tokyo, Japan; and ${ }^{2}$ Department of Neurosurgery, Kawasaki Municipal Hospital, Kanagawa, Japan
}

BACKGROUND The authors' previous cadaveric study reported a new surgical approach that can expose the deep cerebellar hemisphere, cerebellopontine angle, and upper fourth ventricle through dissection of the horizontal fissure of the suboccipital cerebellar hemisphere. Here, the authors present their experience with the first clinical use of the suboccipital trans-horizontal fissure (SOTHF) approach requiring access to the third and upper fourth ventricle lesions, a challenging compartment to access by traditional approaches.

OBSERVATIONS In cases 1 and 2, computed tomography demonstrated large hematomas in the left cerebellar hemisphere with extension into the third ventricle and/or the upper fourth ventricle, resulting in obstructive hydrocephalus. Large hematomas in both the cerebellar hemisphere and the upper fourth ventricle were successfully removed via an SOTHF approach alone without external ventricular drainage. Furthermore, the hematoma in the third ventricle was removed through the aqueduct in case 2 . Access to the upper fourth ventricle and the third ventricle were intraoperatively verified using a neuronavigation system. The patients immediately regained consciousness, and the result of cerebellar function testing was almost normal after the operation.

LESSONS An SOTHF approach can achieve the removal of cerebellar and intraventricular hematomas simultaneously, is a faster and potentially safer method than others, and subsequently allows rapid clinical improvement.

https://thejns.org/doi/abs/10.3171/CASE21647

KEYWORDS cerebellum; hemorrhage; fourth ventricle; posterior cranial fossa; horizontal fissure

We previously reported a novel surgical approach that can expose the deep cerebellar hemisphere, cerebellopontine angle, and upper fourth ventricle through dissection of the horizontal fissure (HF) of the suboccipital cerebellar hemisphere. ${ }^{1}$ In the previous study, the suboccipital trans-HF (SOTHF) approach was compared with other conventional approaches through a cadaveric study. ${ }^{1}$

The approach to the fourth ventricle is challenging because surrounding cerebrovascular structures limit the available working space. Notably, access to the most rostral part of the fourth ventricle and third ventricle is difficult to expose via conventional approaches such as transvermian and transcerebellomedullary fissure
(trans-CMF) approaches. ${ }^{2-4}$ The transvermian approach involves incision of the inferior vermis, which risks cerebellar mutism. ${ }^{4,5}$ Early visualization of the lower fourth ventricle is achieved upon dissection of the CMF immediately, however, to further obtain access to the upper third of the fourth ventricle and, furthermore, the third ventricle, which increases the risk of complications. ${ }^{2,3}$

In the previous cadaveric study, the medial SOTHF approach efficiently allowed visualization of the upper fourth ventricle without incising the inferior vermis, opening the tela choroidea and inferior medullary velum. ${ }^{1}$ The medial SOTHF approach could provide a flatter approach to the upper fourth ventricle, aqueduct, and brainstem.

ABBREVIATIONS CMF = cerebellomedullary fissure; $\mathrm{CT}=$ computed tomography; $\mathrm{GCS}=$ Glasgow Coma Scale; HF = horizontal fissure; ITSA = infratentorial supracerebellar approach; OTA = occipital transtentorial approach; SMV = superior medullary velum; SOTHF = suboccipital transhorizontal fissure.

INCLUDE WHEN CITING Published February 14, 2022; DOI: 10.3171/CASE21647.

SUBMITTED November 13, 2021. ACCEPTED January 3, 2022.

(c) 2022 The authors, CC BY-NC-ND 4.0 (http://creativecommons.org/licenses/by-nc-nd/4.0/). 
Here, we present our experience with the first clinical use of a medial SOTHF approach to the third and upper fourth ventricle lesions.

\section{Illustrative Cases}

\section{Case 1}

A 53-year-old female presented with sudden impairment of consciousness (Glasgow Coma Scale [GCS] score, E1V1M2). Computed tomography (CT) demonstrated a 49-mm-diameter hematoma in the left cerebellar hemisphere with rupture into the fourth ventricle (Fig. 1A). Obstructive hydrocephalus due to the hematoma in the fourth ventricle was observed. The surgical plan was to use the intermediate SOTHF approach and medial SOTHF approach for the hematomas in the cerebellar hemisphere and fourth ventricle, respectively. ${ }^{1}$

The patient was placed in prone position (Fig. 2A). A midline suboccipital incision was used to dissect the soft tissues from the inion to the top of $\mathrm{C} 2$. A suboccipital craniotomy and foramen magnum decompression were performed, and the dura was opened in a Y-shaped fashion. Bony structures covering the left transverse sinus were skeletonized. The suboccipital surface of the HF was observed. First, an intermediate SOTHF approach was planned to remove the cerebellar hematoma (Fig. 2B). The arachnoid membrane of the HF was dissected with a bipolar forceps $2 \mathrm{~cm}$ from the midline to make an intermediate surgical corridor (Fig. 2C). The thick inferior hemispheric vein was not identified in this corridor.
The brain was swollen, and the hematoma was found at a depth of $8 \mathrm{~mm}$ from the cerebellar cortex. The cerebellar hematoma was removed, and the bleeding vessel was coagulated using a bipolar forceps. After sufficient hematoma decompression and confirmation of hemostasis, the evacuated space was filled with artificial cerebrospinal fluid. This large hematoma could be removed without damaging the cerebellar cortex or deep white matter (Fig. 2C).

Next, a medial SOTHF approach was planned to remove the hematoma in the fourth ventricle to relieve obstructive hydrocephalus (Fig. 2D). ${ }^{1}$ Opening the medial HF and creating a medial surgical corridor was performed to expose the superior medullary velum (SMV). Although the surgical route was created medially from the deep cerebellar nuclei, retraction of the superior semilunar lobule was minimized to avoid damaging the deep cerebellar nuclei located within. After medial retraction of both lobules and penetration of the SMV, the upper fourth ventricle packed with hematoma could be visualized directly. Obstructive hydrocephalus was resolved by irrigation of the hematoma within the fourth ventricle (Fig. 2E). DuraGen (Integra Life Sciences Corp.) was inserted intradurally to achieve duraplasty. This surgery took 3 hours, 41 minutes, in total (intermediate SOTHF, 40 minutes; medial SOTHF, 51 minutes; other general procedures, 130 minutes).

A postoperative CT scan displayed complete removal of hematomas in the cerebellar hemisphere and fourth ventricle (Fig. 1A). Improvement of obstructive hydrocephalus was achieved without an external ventricular drainage system. The hematoma cavity was
A
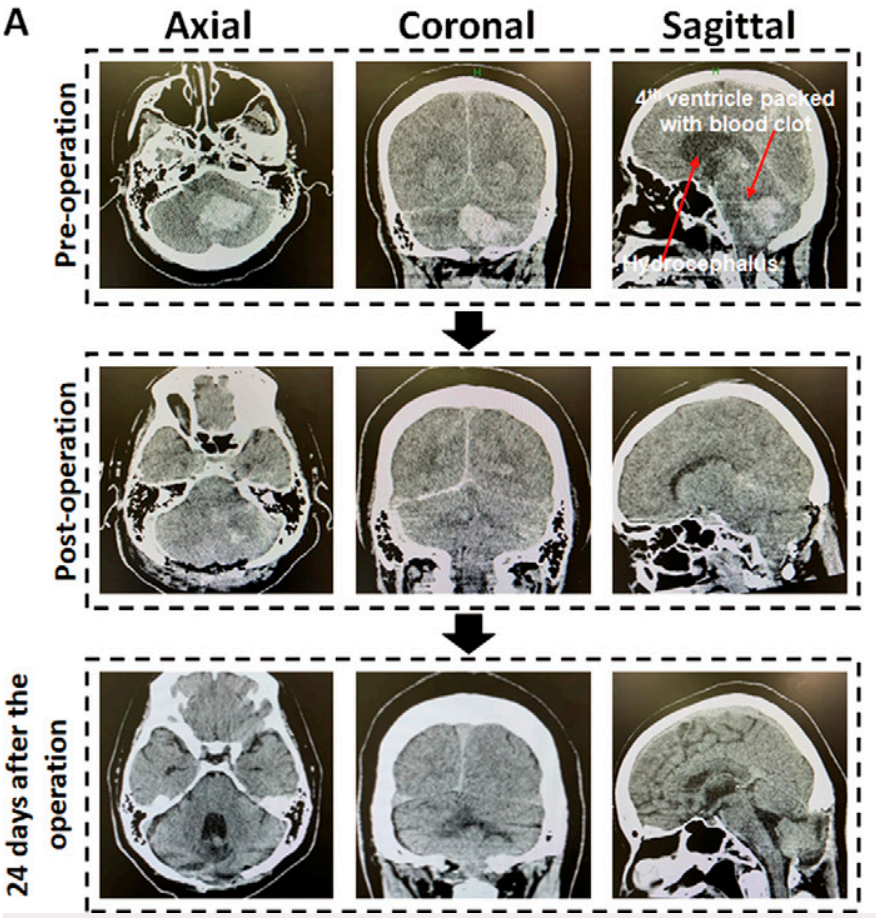

B

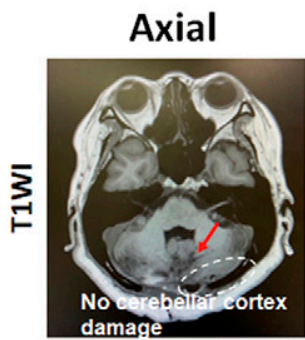

21 days after the operation

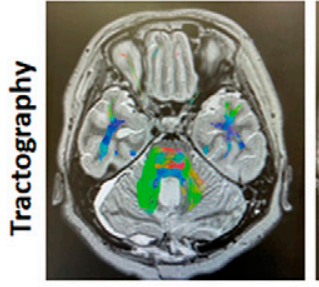
Coronal
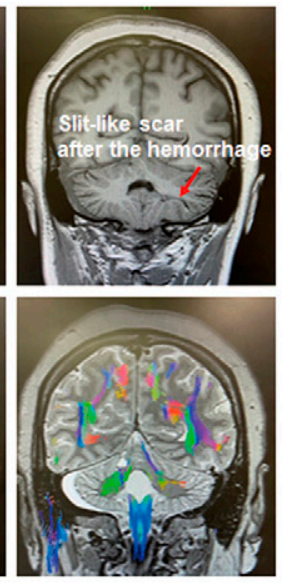

Sagittal
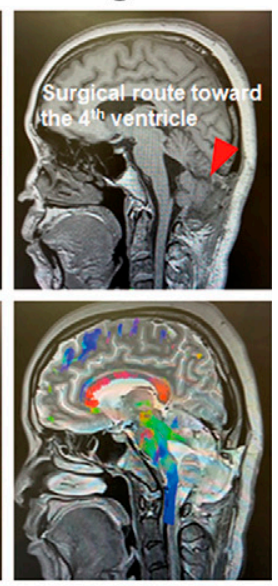

FIG. 1. Pre- and postoperative radiographic findings in case 1. A: CT scan before, 1 day after, and 24 days after the operation (left = axial view; center = coronal view; right = sagittal view). A 49-mm-diameter hemorrhage is shown in the left cerebellar hemisphere, which extends into the fourth ventricle. There is obstructive hydrocephalus due to hematoma in the fourth ventricle. Postoperative CT scan shows complete removal of hematomas in the cerebellar hemisphere and fourth ventricle. Obstructive hydrocephalus is improved without external ventricular drainage. The hematoma cavity is resolved into a slitlike, fluid-filled cavity 24 days after the operation. B: Postoperative magnetic resonance imaging findings 21 days after the operation. T1weighted image (T1WI) shows a slight slitlike cavity in the left cerebellar hemisphere caused by the hemorrhage. No cerebellar cortex damage is present. A surgical route toward upper fourth ventricle is detected on a sagittal view of T1WI. Because the deep cerebellar nuclei are located above the HF, the dentate nucleus can be preserved. In both sides, tractography shows that the superior cerebellar peduncles connect along the dentate nucleus and course anterosuperiorly to exit the cerebellum and continue up the brainstem. 


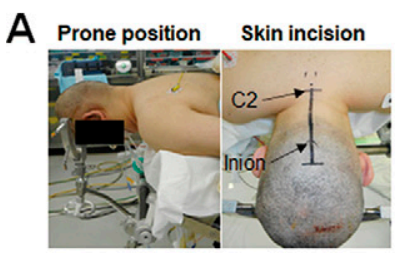

C

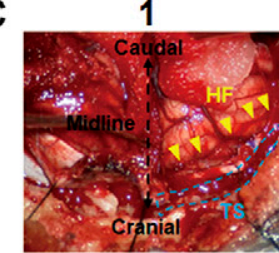

4

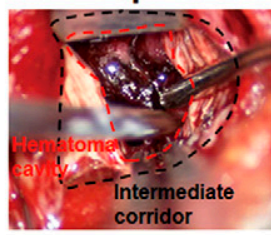

7

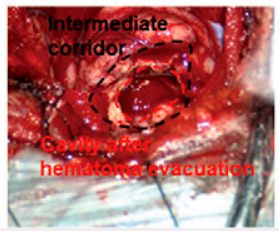

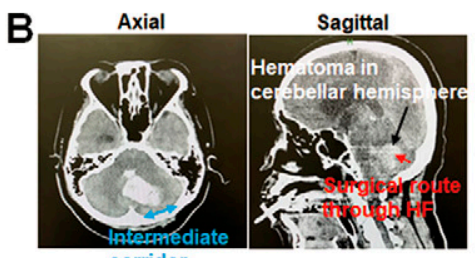

corridor 2

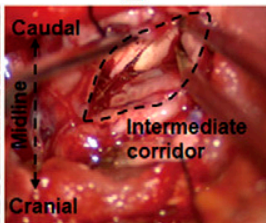

5
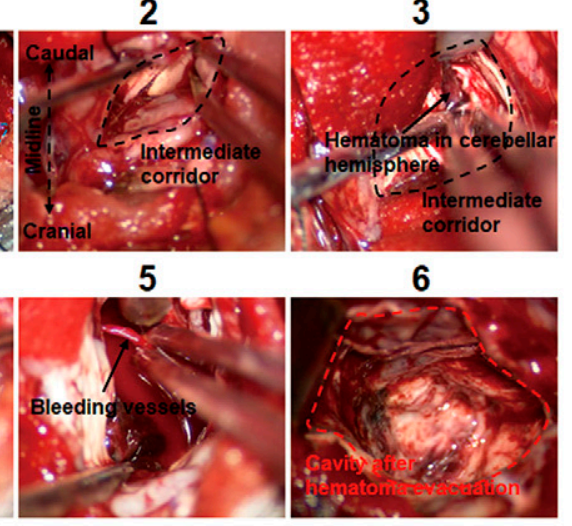

6 .

\section{(}

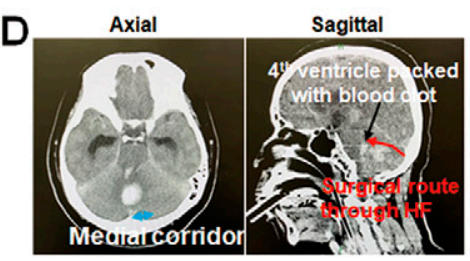

E
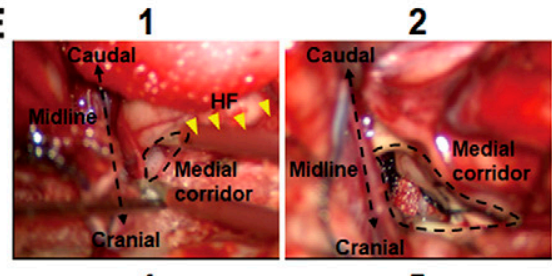

5

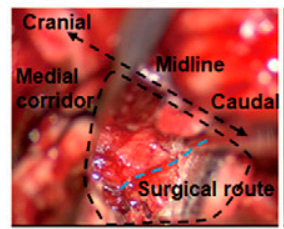

7

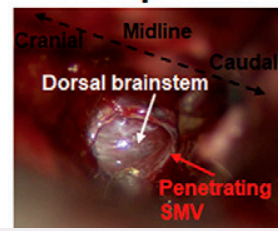

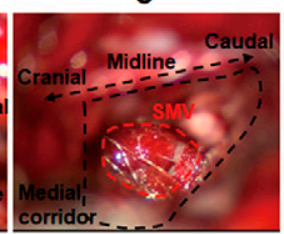

8

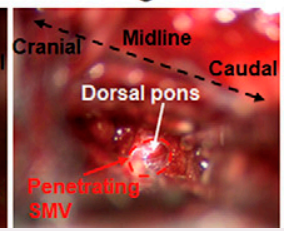

3

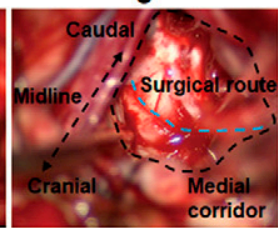

6

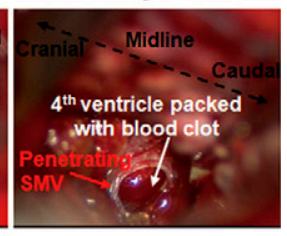

9

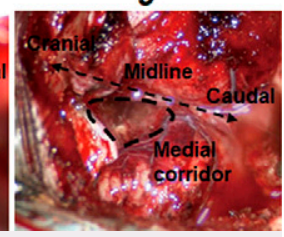

FIG. 2. Intraoperative findings of the intermediate and medial SOTHF approach in case 1. A: The patient is placed in prone position. A midline suboccipital incision is used from the inion to the top of C2. B: Intermediate surgical corridor and surgical route shown on CT scan. C: Microsurgical maneuvers of the intermediate SOTHF approach are shown. Intraoperative photograph after dural opening (1). Left transverse sinus is skeletonized. The left cerebellum is swollen. The HF is the largest and deepest fissure on the suboccipital surface of the cerebellum (yellow arrowhead). The arachnoid membrane of the intermediate HF is dissected with a bipolar forceps (2). No restrictive connective tissue is observed in the fissure (2). The HF of the suboccipital surface is dissected $2 \mathrm{~cm}$ from midline to make an intermediate surgical corridor. The hematoma is found at a depth of $8 \mathrm{~mm}$ from cerebellar cortex $(3,4)$. The bleeding vessel is coagulated using bipolar forceps (5). Large hematoma can be removed without aspiration of cerebellar cortex or deep white matter (6, 7). D: After the intermediate SOTHF approach was completed, medial SOTHF approach was performed to remove hematoma in the upper fourth ventricle and relieve hydrocephalus. Medial surgical corridor and surgical route shown on CT scan. E: Microsurgical maneuvers of the medial SOTHF approach are shown. The arachnoid membrane of the medial HF is dissected with a bipolar forceps $(1,2)$. Medial surgical corridor is made medially from the deep cerebellar nuclei. The operator stands on the transverse sinus or sigmoid sinus side (3, 4). After the medial surgical corridor is made, SMV is clearly identified (5). After the SMV is penetrated, the upper fourth ventricle packed with hematoma can be directly seen (6). Obstructive hydrocephalus is resolved by irrigation of the hematoma in the fourth ventricle. Dorsal brainstem is observed (7). Wide working space can be made by this flatter surgical route $(8,9)$. TS $=$ transverse sinus.

resolved into a slitlike, fluid-filled cavity 24 days after the operation (Fig. 1A). Only a slitlike scar was observed on a T1-weighted image 19 days after the operation (Fig. 1B) and was absent of cortical damage (Fig. 1B). A surgical route toward the upper fourth ventricle was visualized in a sagittal view of a T1-weighted image (Fig. 1B). Notably, the dentate nucleus could be preserved because it is located above the HF. In both sides, tractographies showed that the superior cerebellar peduncles remained connected along the dentate nucleus and coursed anterosuperiorly to exit the cerebellum and continue up the brainstem (Fig. 1B).

Postoperative care was provided as per the standard protocols. The patient completely regained consciousness 5 days after the operation (GCS score, E4V5M6). The findings of cerebellar function testing, including knee pat, dysdiadochokinesia, nose-finger-nose, and heel-shin testing, were almost normal 21 days after the operation. Cerebellar gait, including a widened base, unsteadiness, and irregularity of steps, and lateral veering, although scarcely, were observed.

\section{Case 2}

A 74-year-old male presented with sudden impairment of consciousness (GCS score, E3V2M4). CT demonstrated a 41-mmdiameter hematoma in the left cerebellar hemisphere with rupture into the third and fourth ventricles (Fig. 3). Obstructive hydrocephalus due to the hematoma in the third and fourth ventricles was observed. The surgical plan was to use the intermediate SOTHF approach and medial SOTHF approach for the hematomas in the cerebellar hemisphere and third'fourth ventricles, respectively. ${ }^{1}$

During the intermediate SOTHF approach, the main trunk of the inferior hemispheric vein was preserved; however, a small branch that coursed longitudinally behind the surgical corridor was sacrificed. The arachnoid membrane of the HF was dissected with a bipolar forceps $1.5 \mathrm{~cm}$ from midline to make an intermediate surgical corridor (Fig. 4A). The hematoma was found at a depth of $1 \mathrm{~cm}$ from the cerebellar cortex. Sufficient hematoma decompression and confirmation of hemostasis were achieved without damaging the cerebellar cortex or deep white matter (Fig. 4A). 


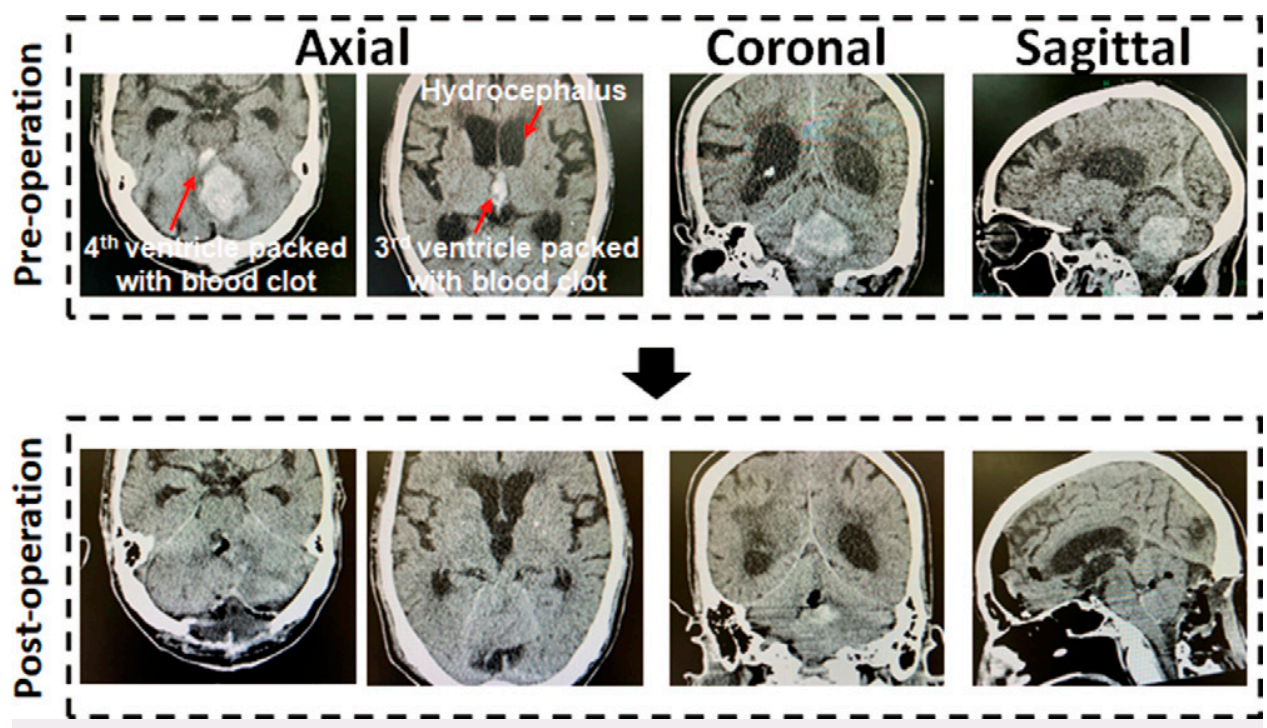

FIG. 3. Pre- and postoperative radiographic findings in case 2. CT scan before and 1 day after the operation (left = axial view; center = coronal view; right = sagittal view). A 41-mm-diameter hemorrhage is shown in the left cerebellar hemisphere, which extends into the third and fourth ventricles. There is obstructive hydrocephalus due to hematomas in the third and fourth ventricles. Postoperative CT scan shows complete removal of hematomas in the cerebellar hemisphere and third and fourth ventricles. Obstructive hydrocephalus is improved without external ventricular drainage.

Next, a medial SOTHF approach was planned to remove the hematoma in the third and fourth ventricles to relieve obstructive hydrocephalus (Fig. 4B). ${ }^{1}$ After medial retraction of both lobules and penetration of the SMV, the upper fourth ventricle packed with hematoma could be visualized directly. The hematomas within the upper and middle fourth ventricles were irrigated (Fig. 4B). The aqueduct was clearly identified, and the third ventricle packed with blood clot was directly visualized through the aqueduct. Obstructive hydrocephalus resolved by hematoma removal in the third ventricle. Structures in the third ventricle, such as the fornix, superior choroidal vein, and choroid plexus, were observed directly through the aqueduct (Video 1). Access to the third and upper fourth ventricles was intraoperatively verified using a neuronavigation system (BrainLab) (Fig. 4C). DuraGen was inserted intradurally to achieve duraplasty. This surgery took 2 hours, 42 minutes in total (intermediate SOTHF, 20 minutes; medial SOTHF, 32 minutes; other general procedures, 110 minutes).

VIDEO 1. Clip showing intermediate SOTHF and medial SOTHF approaches for the hematomas in the cerebellar hemisphere and upper fourth and third ventricles (related to Fig. 4). Combination of the intermediate SOTHF and medial SOTHF approaches can accomplish the simultaneous removal of hematomas in the cerebellar hemisphere and upper fourth and third ventricles in case 2. Click here to view.

A postoperative CT scan displayed complete removal of hematomas in the cerebellar hemisphere and third and fourth ventricles (Fig. 3). Improvement of obstructive hydrocephalus was achieved without an external ventricular drainage system.

The patient immediately regained consciousness after the operation (GCS score, E4V5M6). Cerebellar function tests were almost normal after the operation.

\section{Step-by-Step Explanation of Approaches} Intermediate SOTHF Approach

Making the Intermediate Corridor

The lesion is accessed starting with dissection of the HF (toward the pathology; Fig. 5A). A large, deep-seated hematoma is removed without additional sacrifice of the cerebellar cortex or deep white matter (Fig. 5C).

\section{Medial SOTHF Approach}

Dissecting the Arachnoid Membrane and Cortex

A medial surgical corridor is created by opening the medial side of the HF (1.5 cm from the midline; Fig. $5 \mathrm{~A}, \mathrm{~B}$, and D). The arachnoid membrane and underlying cortex between the cerebellar hemisphere and vermis are dissected (Fig. 5E).

\section{Dissecting the White Matter}

A dissection line between the cerebellar hemisphere and the vermis along the white matter fibers is created in a blunt manner. The SMV can be recognized by its veering plane upward along with the fibers. The superior cerebellar peduncle is visualized toward the lateral side of this process (Fig. $5 \mathrm{E}$ ).

\section{Penetrating the SMV}

The SMV, which is located at the end of this corridor, can be recognized by its whitish appearance. A 5-10-mm caudal incision allows penetration into the upper fourth ventricle. The hematoma packed in the upper fourth ventricle can be directly removed (Fig. 5E).

\section{Identifying the Aqueduct and Third Ventricle}

The aqueduct can be identified by directing the microscope superomedially. Obstructive hydrocephalus is resolved through the aqueduct by irrigation of the hematoma in the third ventricle (Fig. 5E). 
A

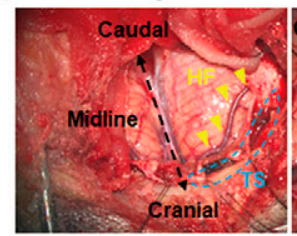

4

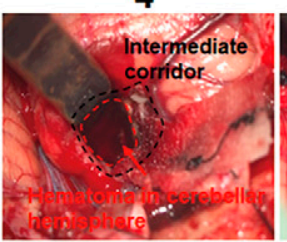

c

Axial
2

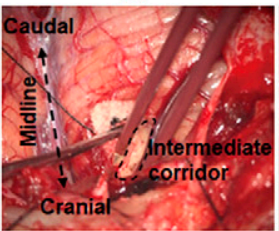

5
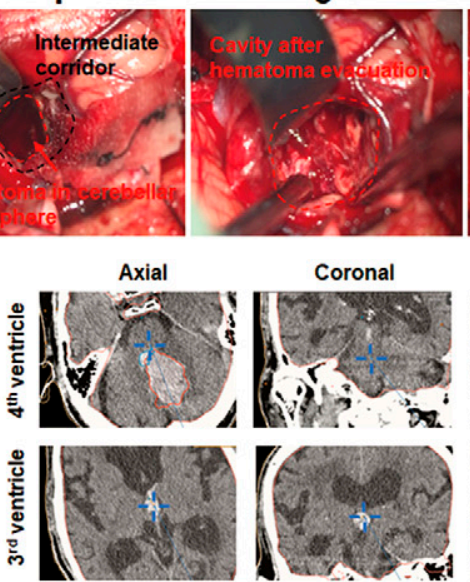

3

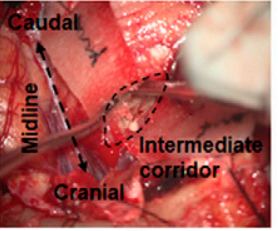

6

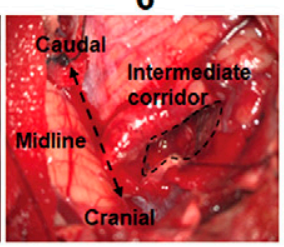

Sagittal

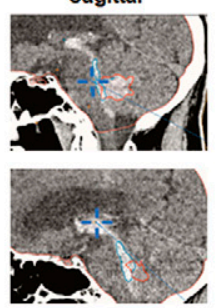

B

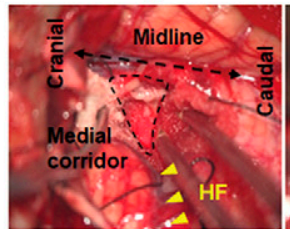

4

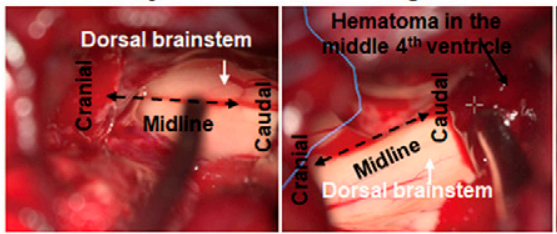

7

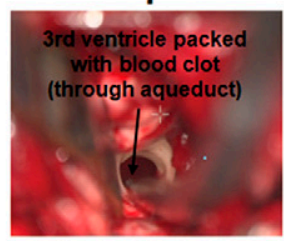

2

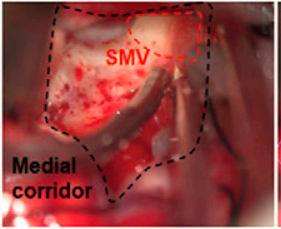

5

8

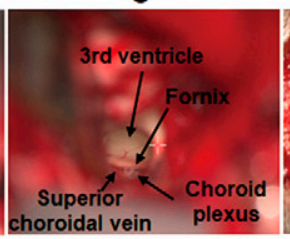

3

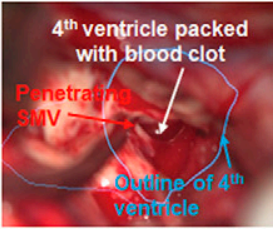

6

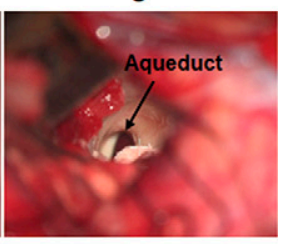

9

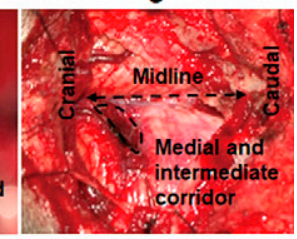

FIG. 4. Intraoperative findings of the intermediate and medial SOTHF approach in case 2. A: Microsurgical maneuvers of the intermediate SOTHF approach are shown. Intraoperative photograph after dural opening (1). Left transverse sinus is skeletonized. The HF is the largest and deepest fissure on the suboccipital surface of the cerebellum (yellow arrowheads). The arachnoid membrane of the intermediate HF is dissected with a bipolar forceps. Inferior hemispheric vein is preserved (2). The HF of the suboccipital surface is dissected $1.5 \mathrm{~cm}$ from midline to make an intermediate surgical corridor. The hematoma is found at a depth of $1 \mathrm{~cm}$ from cerebellar cortex $(3,4)$. Large hematoma can be removed through intermediate corridor without aspiration of cerebellar cortex or deep white matter $(5,6)$. B: After the intermediate SOTHF approach was completed, medial SOTHF approach was performed to remove hematoma in the third ventricle and upper fourth ventricle. Microsurgical maneuvers of the medial SOTHF approach are shown. The arachnoid membrane of the medial HF is dissected with a bipolar forceps. No restrictive connective tissue is observed in the fissure (1). The operator stands on the transverse sinus or sigmoid sinus side. After creation of a medial surgical corridor, SMV is clearly identified (2). After penetration of the SMV, the upper fourth ventricle packed with hematoma can be directly visualized (3). Hematoma in the upper fourth ventricle is removed. Dorsal brainstem is observed (4). Hematoma in the middle fourth ventricle is removed (caudal microscope direction) (5). Aqueduct is clearly identified (cranial microscope direction) (6). The third ventricle packed with blood clot is seen through aqueduct (7). Obstructive hydrocephalus is resolved by irrigation of the hematoma in the third and fourth ventricles. Fornix, superior choroidal vein, and choroid plexus are seen in the third ventricle (8). Wide working space can be made by the medial and intermediate SOTHF approaches (9). C: Access to the third and upper fourth ventricles is intraoperatively verified using neuronavigation system. $\mathrm{TS}=$ transverse sinus.

\section{Discussion}

\section{Observations}

In general, patients with cerebellar hemorrhages greater than 3 $\mathrm{cm}$ in diameter with neurological deterioration or who have brainstem compression and hydrocephalus from ventricular obstruction are recommended to have surgical removal of the hematoma and the use of external drainage devices to relieve hydrocephalus. ${ }^{6}$ However, external drainage devices must be checked regularly, and, because of infectious complications, the duration of placement should not exceed 7 days. ${ }^{6,7}$ Furthermore, early aggressive rehabilitation is recommended in many guidelines. Initiation of rehabilitative strategies within the first 2 weeks of stroke contribute to beneficial outcomes, preventing negative effects on the musculoskeletal, cardiovascular, respiratory, and immune systems and reducing immobility-related complications. ${ }^{8}$ However, early aggressive rehabilitation cannot be performed for the patients with external drainage devices.

In these patients, an intermediate and medial SOTHF approach could simultaneously remove hematomas in the cerebellum and fourth ventricle. Furthermore, the hematoma in the third ventricle was successfully removed through the aqueduct during the medial SOTHF approach, leading to rapid improvement of cerebellar swelling and hydrocephalus without an external drainage device. An SOTHF approach could prevent the risk of ventricular drainage-related infection and prompt early aggressive rehabilitation.

An infratentorial supracerebellar approach (ITSA) and an occipital transtentorial approach (OTA) are used for posterior third ventricle lesions. ITSA provides optimal exposure for lesions in the pineal region, posterior third ventricle, and posterior mesencephalon. However, the ITSA has limitations when the lesion shows lateral or caudal extension. Furthermore, it is contraindicated in patients with a steeply angled tentorium. ${ }^{9}$ The OTA provides access for the removal of lesions involving the splenium, pineal gland, collicular plate, cerebellomesencephalic fissure, and anterosuperior part of the cerebellum. Some disadvantages of OTA include the risk of damage to the occipital lobe or internal occipital vein, risk of damage to deep vein structures, and poor visualization of the contralateral quadrigeminal region. ${ }^{10}$

Furthermore, rigid and flexible endoscopic procedures can be used through a single bur hole for patients with fourth ventricle and 

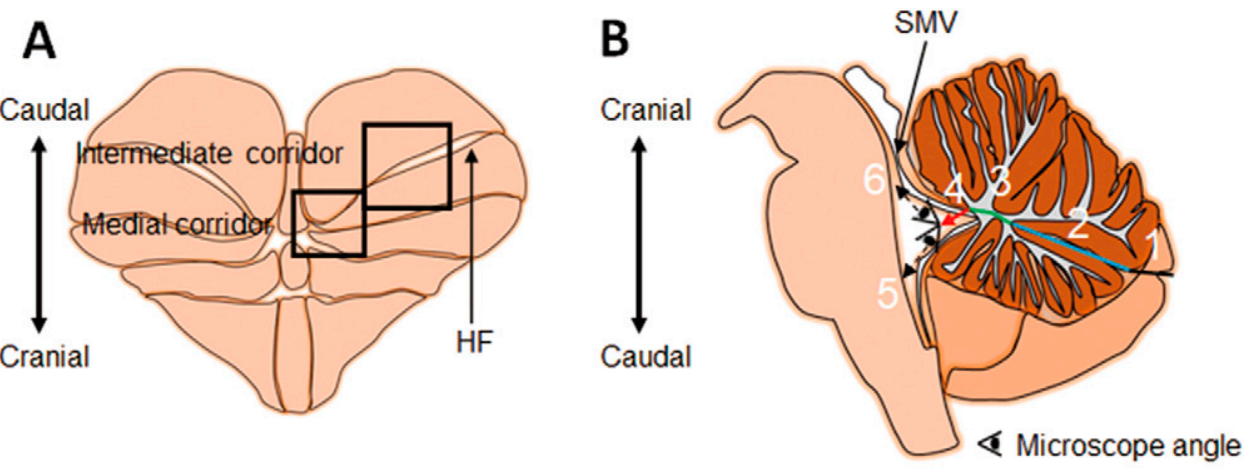

C Intermediate SOTHF

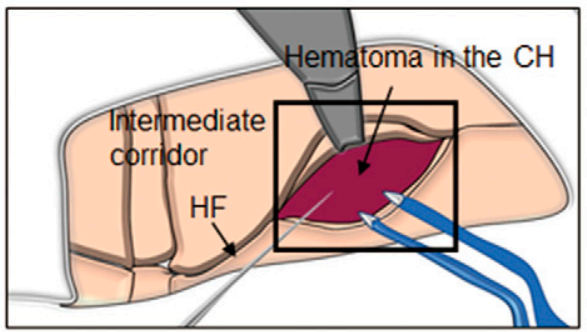

D Medial SOTHF
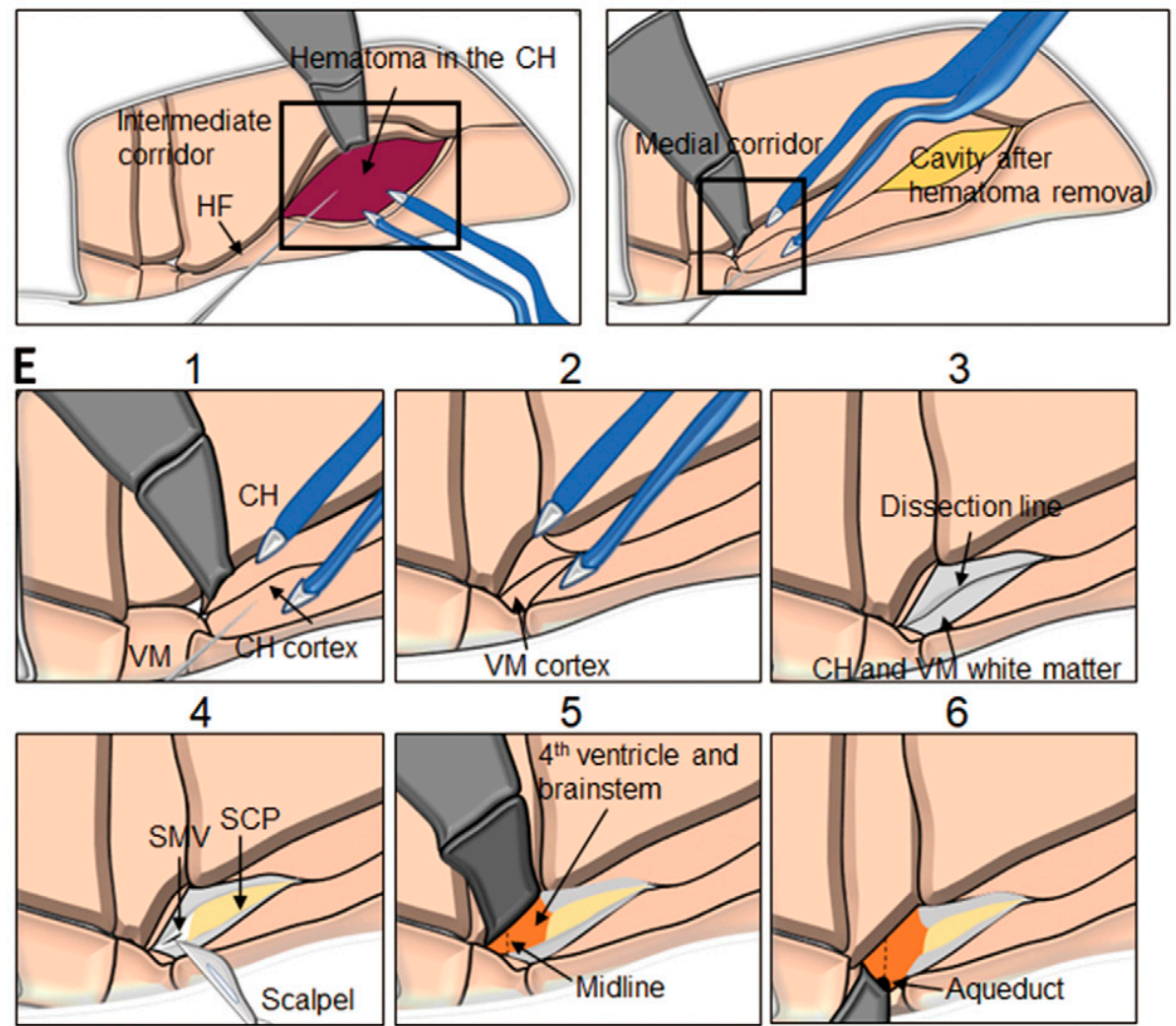

FIG. 5. Step-by-step explanation of this approach. A: Posterior view of a cerebellum before HF dissection. Intermediate and medial corridors are shown. B: Midline sagittal view of cerebellum and brainstem. C: Intermediate SOTHF approach is shown. The cerebellar hematoma can be removed through the intermediate corridor. D: Medial SOTHF approach is performed to remove the hematoma in the third and fourth ventricles. A medial surgical corridor is created by opening the medial side of the HF (1.5 cm from the midline). E: Arachnoid membrane and underlying cortex between the cerebellar hemisphere and vermis are dissected $(1,2)$. A dissection line between the cerebellar hemisphere and the vermis along the white matter fibers is created in a blunt manner (3). The SMV can be recognized by its veering plane upward along with the fibers. The superior cerebellar peduncle is visualized toward the lateral side of this process (4). The SMV, which is located at the end of this corridor, can be recognized by its whitish appearance. A 5-10-mm caudal incision allows penetration into the upper fourth ventricle. The hematoma packed in the upper fourth ventricle can be directly removed (5). The aqueduct can be identified by directing the microscope superomedially. Obstructive hydrocephalus was resolved through aqueduct by irrigation of the hematoma in the third ventricle (6). $\mathrm{CH}=$ cerebellar hemisphere; SCP = superior cerebellar peduncle; VM = vermis.

posterior third ventricle lesions. In particular, the flexible endoscope enables easy access to posterior third ventricle lesions (e.g., pineal, aqueductal, and tectal tumors and select cases of fourth ventricle tumors) without imposing excessive pressure on the foramen of Monro. ${ }^{11}$ Additionally, rigid scopes can be used for inspecting the aqueductal entry and performing balloon aqueductoplasty. ${ }^{12,13}$ 
Although the use of neuroendoscopy has increased in modern neurosurgery, one disadvantage of the endoscope is the restricted ability to maneuver the instrument.

The medial SOTHF approach efficiently provides thorough surgical exposure and meticulous operability of the upper fourth ventricle without incising the inferior vermis and opening the tela choroidea and inferior medullary velum. The medial SOTHF approach provides a flatter approach to the upper fourth ventricle and brainstem. Although lesions around the third ventricle, upper fourth ventricle, and cerebellar hemisphere cannot be exposed simultaneously via conventional approaches, a medial SOTHF approach can give direct access to these lesions simultaneously. This approach did not require a longer operative time than previously reported conventional approaches for cerebellar hemorrhages. ${ }^{14}$ After the removal of the cerebellar hematoma through the intermediate corridor, it took 32-51 minutes to irrigate the third and upper fourth ventricles through the medial corridor. A flatter surgical route can shorten the operative time to expose the structures around the third and upper fourth ventricles.

In this case, the SOTHF approach to the third and fourth ventricles might have been facilitated by the expansion of the corridor made by the hematoma. In particular, access to the third ventricle depends on the size of the aqueduct. Therefore, the combination with a neuroendoscope may be needed to sufficiently cover the posterior third ventricle. This approach needs to be further assessed for other isolated third and fourth ventricle pathologies. Furthermore, the combination of medial SOTHF and ITSA/OTA will allow coverage of a broad area around the upper fourth ventricle and posterior third ventricle. Further studies are warranted to confirm the merits of this combined strategy.

\section{Lessons}

Combination of the intermediate SOTHF and medial SOTHF approaches can accomplish the simultaneous removal of hematomas in the cerebellar hemisphere and upper fourth and third ventricles, respectively.

\section{References}

1. Tamura R, Katayama M, Yamamoto K, Horiguchi T. Suboccipital trans-horizontal fissure approach for posterior cranial fossa lesions: a cadaveric study and first clinical experience. Oper Neurosurg (Hagerstown). 2021;21(6):E479-E487.

2. Gök A, Alptekin M, Erkutlu I. Surgical approach to the fourth ventricle cavity through the cerebellomedullary fissure. Neurosurg Rev. 2004;27(1):50-54.

3. Matsushima T, Inoue T, Inamura T, Natori Y, Ikezaki K, Fukui M. Transcerebellomedullary fissure approach with special reference to methods of dissecting the fissure. J Neurosurg. 2001;94(2):257-264.

4. Tanriover N, UIm AJ, Rhoton AL Jr, Yasuda A. Comparison of the transvermian and telovelar approaches to the fourth ventricle. $J$ Neurosurg. 2004;101(3):484-498.
5. Pollack IF, Polinko P, Albright AL, Towbin R, Fitz C. Mutism and pseudobulbar symptoms after resection of posterior fossa tumors in children: incidence and pathophysiology. Neurosurgery. 1995;37(5): 885-893.

6. Broderick JP, Adams HP Jr, Barsan W, et al. Guidelines for the management of spontaneous intracerebral hemorrhage: a statement for healthcare professionals from a special writing group of the Stroke Council, American Heart Association. Stroke. 1999;30(4): 905-915.

7. Hoefnagel D, Dammers R, Ter Laak-Poort MP, Avezaat CJ. Risk factors for infections related to external ventricular drainage. Acta Neurochir (Wien). 2008;150(3):209-214.

8. Bernhardt J, Godecke E, Johnson L, Langhorne P. Early rehabilitation after stroke. Curr Opin Neurol. 2017;30(1):48-54.

9. Oliveira J, Cerejo A, Silva PS, Polónia P, Pereira J, Vaz R. The infratentorial supracerebellar approach in surgery of lesions of the pineal region. Surg Neurol Int. 2013;4:154.

10. Yamamoto I. Pineal region tumor: surgical anatomy and approach. J Neurooncol. 2001;54(3):263-275.

11. Roth J, Constantini S. Combined rigid and flexible endoscopy for tumors in the posterior third ventricle. J Neurosurg. 2015;122(6): 1341-1346.

12. Schroeder HW, Gaab MR. Endoscopic aqueductoplasty: technique and results. Neurosurgery. 1999;45(3):508-518.

13. Toyota S, Taki T, Oshino S, et al. A neuroendoscopic approach to the aqueduct via the fourth ventricle combined with suboccipital craniectomy. Minim Invasive Neurosurg. 2004;47(5):312-315.

14. Tamaki T, Kitamura T, Node Y, Teramoto A. Paramedian suboccipital mini-craniectomy for evacuation of spontaneous cerebellar hemorrhage. Neurol Med Chir (Tokyo). 2004;44(11):578-583.

\section{Disclosures}

The authors report no conflict of interest concerning the materials or methods used in this study or the findings specified in this paper.

\section{Author Contributions}

Conception and design: Tamura, Katayama, Horiguchi. Acquisition of data: Tamura, Kuranari. Analysis and interpretation of data: Tamura, Horiguchi. Drafting the article: Tamura, Katayama. Critically revising the article: Katayama, Kuranari. Reviewed submitted version of manuscript: Horiguchi. Approved the final version of the manuscript on behalf of all authors: Tamura. Administrative/technical/material support: Kuranari, Horiguchi. Study supervision: Horiguchi.

\section{Supplemental Information \\ Videos}

Video 1. https://vimeo.com/662924849.

\section{Correspondence}

Ryota Tamura: Keio University School of Medicine, Tokyo, Japan. moltobello-r-610@keio.jp. 